\author{
B. J. Huang \\ Y. C. Chen \\ Department of Mechanical Engineering, \\ National Taiwan University, \\ Taipei, Taiwan
}

\section{System Dynamics and Control of a Linear Compressor for Stroke and Frequency Adjustment}

This paper presents results of an investigation on the system dynamics and the controller design of a linear compressor for stroke and frequency adjustment. A system dynamics model was derived and identified experimentally. A control system was designed based on the system dynamics model. The control system used a PDF (Pseudo-DerivativeFeedback) algorithm. The results of step response test for stroke regulation show that, it takes about $0.2 \mathrm{~s}$ for the regulation with small overshoot and negligible steady-state error. For the step change of operating frequency, it takes about 0.4 s to reach steady state with small tracking error. The control system also has very good performance for disturbance rejection. The transient periods are about $0.2 \mathrm{~s}$ with stroke variation to within $10 \%$. The controller is shown to have the capability to replace the conventional crank-shaft mechanism as in a reciprocating compressor. The controller can also be used to regulate both stroke and frequency of the compressor during operation. The performance can thus be very flexible and efficient for a system using the linear compressor and the present control system. [DOI: 10.1115/1.1433802]

\section{Introduction}

The traditional reciprocating-type compressor uses a crankshaft mechanism to convert the rotating motion of an electric motor into reciprocating motion to drive a piston. Cadman and Cohen $[1,2]$ developed a linear compressor in order to eliminate the common problems of wear, vibration and noise due to the side forces exerted on the piston and cylinder wall in a reciprocating-type compressor. A linear compressor utilizes a linear motor to directly drive the piston in unidirectional motion. A well-designed linear compressor thus can greatly reduce side forces and wear, as well as vibration and noise.

Linear compressors have been utilized in many applications, mostly in small refrigerators, air and water pumps, etc. A recent application is in miniature cryocoolers such as the Stirling cooler used as a gas compressor (de Jonge and Sereny [3], Barry [4], Stolfi and Daniels [5]). However, a linear compressor also has some drawbacks. Due to the electromechanical effect, the stroke of the piston (maximum displacement) in a linear compressor varies markedly with the outlet pressure variation. A slight increase in outlet pressure will cause a dramatic decrease in stroke. The performance of a refrigerator or air or water pump using a linear compressor is thus not very good when operating under variable conditions. This indicates that, in order to obtain a fixed piston stroke as in a conventional reciprocating compressor, a linear compressor requires an additional device that can replace the conventional crank-shaft mechanism and have the function of fixing the stroke.

The purpose of the present study is to develop an analog control device that can control the piston stroke of a linear compressor to a desired value. The operating frequency of the linear compressor can also be controlled simultaneously so that it performs just like a variable-frequency compressor. Using such a control system, we can simultaneously control both stroke and frequency thus greatly increasing the flexibility in the control of a refrigerator using a linear compressor. Before attempting a control system design, the system dynamics model of a linear compressor will be studied first.

Contributed by the Dynamic Systems and Control Division for publication in the JouRnal OF DYNAMIC Systems, MEASUREMENT, AND CONTROL. Manuscript received by the Dynamic Systems and Control Division February 28, 2001. Associate Editor: F. Conrad.

\section{System Dynamics Model of a Linear Compressor}

Modeling of a Linear Compressor. Figure 1 illustrates the schematic of a linear compressor. Applying an AC current to the coil, which is placed in a magnetic field, the coil/piston component will move back and forth along the axial direction due to the electromagnetic effect.

The dynamics model of the linear compressor can be derived from the governing equations of electromagnetic behavior and mechanical force balance (Cadman and Cohen [1,2], de Jonge and Sereny [3]). The electromagnetic relation for a linear motor can be expressed as

$$
V_{a}=L_{a} \frac{d I_{a}}{d t}+R_{a} I_{a}+B l_{e} \frac{d X}{d t}
$$

where $V_{a}$ is the driving voltage of the linear motor; $I_{a}$ is the driving current; $L_{a}$ and $R_{a}$ are the inductance and the resistance of the coil, respectively; $B$ is the magnetic flux density in the air gap between the stator and the moving coil; $X$ is the displacement of the piston; $l_{e}$ is the effective length of the coil. The last term in the right-hand side of Eq. (1), $B l_{e} d X / d t$ represents the counter electromotive force according to Faraday's law.

The mechanical force balance of the moving coil and piston results in the following equation:

$$
M \frac{d^{2} X}{d t^{2}}+C \frac{d X}{d t}+K X=l_{e} I_{a} B-A_{p} \Delta P
$$

where $M$ is the equivalent mass of the moving coil and piston, $C$ the viscous damping coefficient, $K$ the spring constant, $A_{p}$ the cross-sectional area of the piston, $\Delta P$ the pressure difference between the compression chamber and the backside of the piston. Laplace transforming to the above equations, we obtain a system dynamics model of the linear compressor:

$$
X(s)=G(s) V_{a}(s)+W(s) \Delta P(s)
$$

where

$$
G(s)=\frac{B l_{e}}{M L_{a} s^{3}+\left(M R_{a}+C L_{a}\right) s^{2}+\left(C R_{a}+B^{2} l_{e}^{2}+L_{a} K\right) s+R_{a} K}
$$




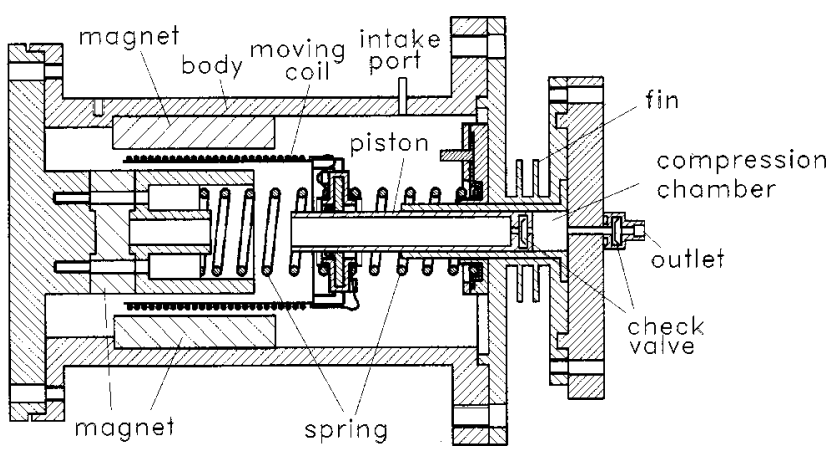

Fig. 1 Structure of the linear compressor

$$
\begin{aligned}
W(s)= & \\
& -\frac{\left(L_{a} s+R_{a}\right) A_{p}}{M L_{a} s^{3}+\left(M R_{a}+C L_{a}\right) s^{2}+\left(C R_{a}+B^{2} l_{e}^{2}+L_{a} K\right) s+R_{a} K}
\end{aligned}
$$

The last term $W(s) \Delta P(s)$ in Eq. (3) represents the effect of disturbance due to the variation of the pressure difference $\Delta P$, while the term $G(s) V_{a}(s)$ represents the response of piston displacement caused by the driving voltage $V_{a}$. The dynamic model of the linear compressor for the control system design can thus be defined with respect to the driving voltage, i.e.,

Table 1 Design specifications of the linear compressor

\begin{tabular}{ll}
\hline total mass of coil/piston $M$ & $0.186 \mathrm{~kg}$ \\
piston diameter $d_{p}$ & $11 \mathrm{~mm}$ \\
diameter of wounded coil & $43 \mathrm{~mm}$ \\
coil wire diameter & $0.7 \mathrm{~mm}$ \\
coil wire length $l_{e}$ & $36 \mathrm{~m}$ \\
inductance of coil $L_{a}$ & $0.0165 \mathrm{Henry}$ \\
electric resistance of coil $R_{a}$ & $3.278 \mathrm{Ohm}$ \\
magnetic flux density $B$ & $0.2 \mathrm{Tesla}$ \\
spring constant $K$ & $5226 \mathrm{~N} / \mathrm{m}$ \\
\hline
\end{tabular}

$$
\begin{aligned}
G(s) & =\frac{X(s)}{V_{a}(s)} \\
& =\frac{B l_{e}}{M L_{a} s^{3}+\left(M R_{a}+C L_{a}\right) s^{2}+\left(C R_{a}+B^{2} l_{e}^{2}+L_{a} K\right) s+R_{a} K}
\end{aligned}
$$

A control system should possess not only a good dynamic response with respect to the reference command of stroke, but also shows robustness in the rejection of disturbances in the variation of pressure difference $\Delta P$.

System Identification of $\boldsymbol{G}(s)$. The dynamic model of a linear compressor, Eq. (6), is theoretical. Some parameters defined in the model can be accurately identified from experiment. A linear compressor was designed and manufactured in the laboratory. The design specifications are given in Table 1. An apparatus designed for the system identification is shown in Fig. 2. A PRBS (pseudorandom-binary signal) input (driving voltage $V_{a}$ ) is used to drive the linear compressor and the response of piston displacement $X(t)$ is measured by a LVDT (linear variable-differential transducer). The PRBS signal is generated by a PC digitally using 11 shift registers and clock period $0.005 \mathrm{~s}$. The digital PRBS signal is converted into an analog signal by a PCL-728 D/A card. A power amplifier finally amplifies the analog signal in order to drive the linear compressor.

The input and output signals of the linear compressor, $V_{a}(t)$ and $X(t)$, are sampled by a signal analyzer (YEW3655E) and recorded by the PC via an IEEE488 interface. The frequency response of the linear compressor $G(j \omega)$ is determined in the relation:

$$
G(j \omega)=\frac{S_{V X}(j \omega)}{S_{V V}(j \omega)}
$$

where $S_{V V}(j \omega)$ is the auto-spectral density function of the input signal $V_{a}(t)$ and $S_{V X}(j \omega)$ is the cross-spectral density function of $V_{a}(t)$ and $X(t)$. For convenience, the dynamic model of the linear compressor can be expressed as

$$
G(s)=\frac{k e^{-\tau_{d}}}{\left(s+p_{1}\right)\left(s+p_{2}\right)\left(s+p_{3}\right)},
$$

where $-p_{1}, \quad-p_{2}, \quad-p_{3}$ are the poles, $k$ is the steady-state gain and $\tau_{d}$ is the time delay accounting for un-modeled dynamics.

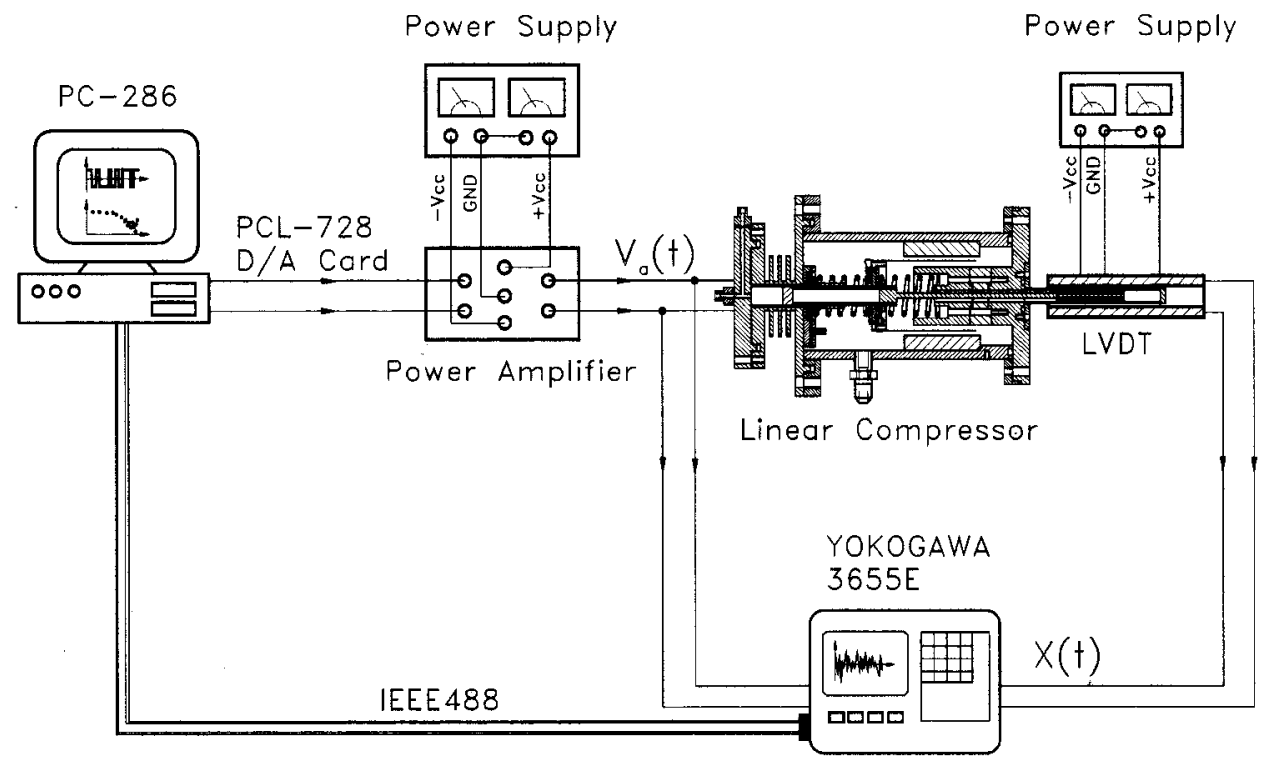

Fig. 2 Experimental apparatus for system identification 

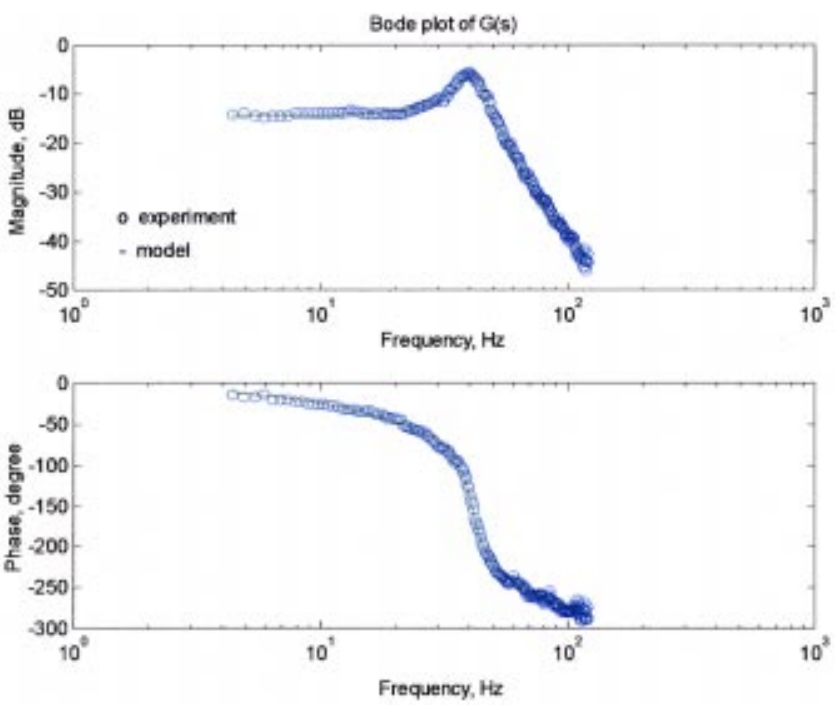

Fig. 3 Frequency response for $G(s)$

The measured $G(j \omega)$ is shown in Fig. 3 which agrees with the model prediction using the identified parameters: $k=2.4876$ $\times 10^{6} ; \quad p_{1}=+188.59 ; \quad p_{2}=+29.69+257.78 j ; \quad p_{3}=+29.69$ $-257.78 j ; \tau_{d}=0.0007 \mathrm{~s}$. Figure 4 shows the input and output signals measured and model predicted. The above results are obtained using the PRBS input $V_{a}(t)$ at $\pm 10 \mathrm{~V}$. Similar dynamic testing were also carried out for $\pm 15 \mathrm{~V}$ and $\pm 20 \mathrm{~V}$ inputs, with the identified parameters almost identical, as shown in Table 2. This indicates that the linear compressor is linear in system dynamics behavior. It is also shown from Fig. 5 that, the experimental results coincide with the theoretical model, Eq. (6), with the design parameters presented in Table 1 and choosing $C=5 \mathrm{~N} \mathrm{~s} / \mathrm{m}$.

\section{Control System Design}

Control System Structure. The control of the stroke and phase of the piston in a linear compressor requires a much more complicated technique. Yang and Huang [6] developed a microprocessor-based fuzzy control system for a linear compressor for a split-type free-displacer Stirling cryocooler. Both stroke and phase of the piston as well as the displacer motion can be
Table 2 Identified system parameters under different inputs

\begin{tabular}{c|c|c|c|c|}
\hline Input, $V_{a}$ & $K, \mathrm{~mm} / V$ & $p_{l}$ & $p_{2}, p_{3}$ & $\tau_{d}, s$ \\
\hline \pm 10 & $2.4876 \times 10^{6}$ & 188.59 & $29.69 \pm j 257.78$ & 0.0007 \\
\hline \pm 15 & $2.4131 \times 10^{6}$ & 179.24 & $30.56 \pm j 258.50$ & 0.0007 \\
\hline \pm 20 & $2.3294 \times 10^{6}$ & 176.61 & $29.78 \pm j 258.16$ & 0.0007 \\
\hline
\end{tabular}

controlled in order to achieve the optimum phase adjustment between the piston and the displacer in Stirling cryocooler.

For air-conditioning and refrigeration or some other applications, the phase control in piston motion is not of primary importance and the control system can be simplified. An analog control system is thus developed as shown in Fig. 6. A DC/AC converter is placed after the stroke controller in order to convert a DC signal into $\mathrm{AC}$ power for driving the linear compressor. The frequency of the AC power signal is adjusted by the frequency setting device that consists of a sine wave function generator. The displacement of the piston $X(t)$ is measured by a LVDT and the signal is processed by a stroke measuring device that converts the time-variant signal of displacement directly into a DC signal $E_{d c}$ representing the magnitude of stroke of the piston. The desired stroke $S$ $\left(=2 X_{r}\right)$ is set in the stroke setting device that converts the command into a DC analog signal, which is compared with the feedback signal $E_{d c}$. The stroke controller picks up the error $E 1$ $=V_{r}-E_{d c}$ and gives an output E2 according to the controller scheme.

The analog control system shown in Fig. 6 eliminates the phase tracking of the piston motion and simplifies the control system a great deal. Yet, the operating frequency of the linear compressor can still be adjusted simultaneously by regulating the frequency setting value.

PDF Controller Design. The control system design follows the feedback design shown in Figure 6. The implementation of the control system is shown in Fig. 7. The DC/AC signal converter consists of a 4-quadrant multiplier that mixes the two inputs and generates a sine wave signal with amplitude in accordance to the controller output signal E2.

A PDF(Pseudo-Derivative-Feedback) controller is used in the present design. The PDF controller has flexibility in design and also reveals better robustness in disturbance rejection (Phelan [7], Leu and Freed [8], Kang et al. [9]). The system dynamics of the linear compressor $G(s)$ presented in Fig. 5 shows that the bandwidth is around $60 \mathrm{~Hz}$. However, the controlled plant $H(s)$ in-
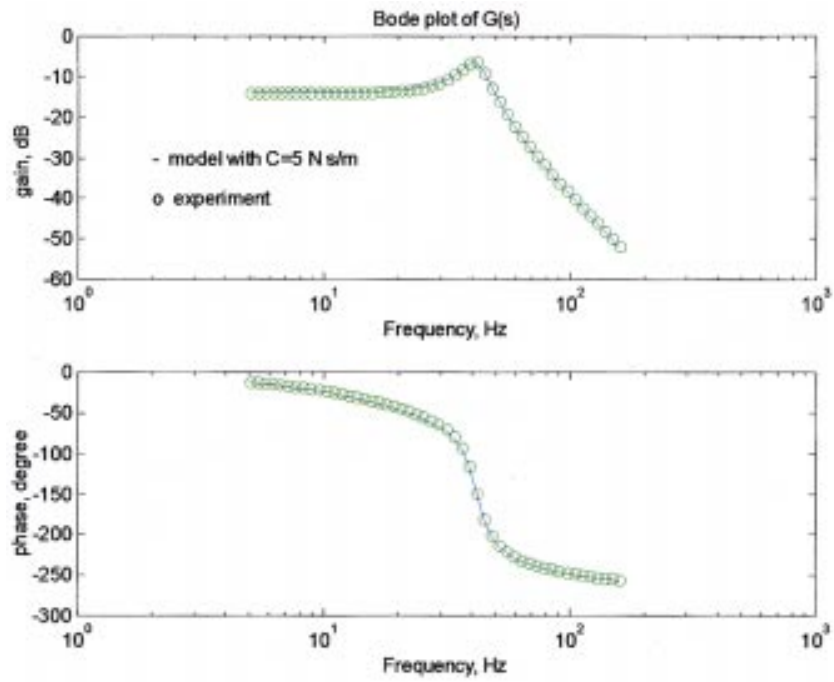

Fig. 5 Theoretical model and experimental results of $G(s)$

Fig. 4 Input and time response of displacement 


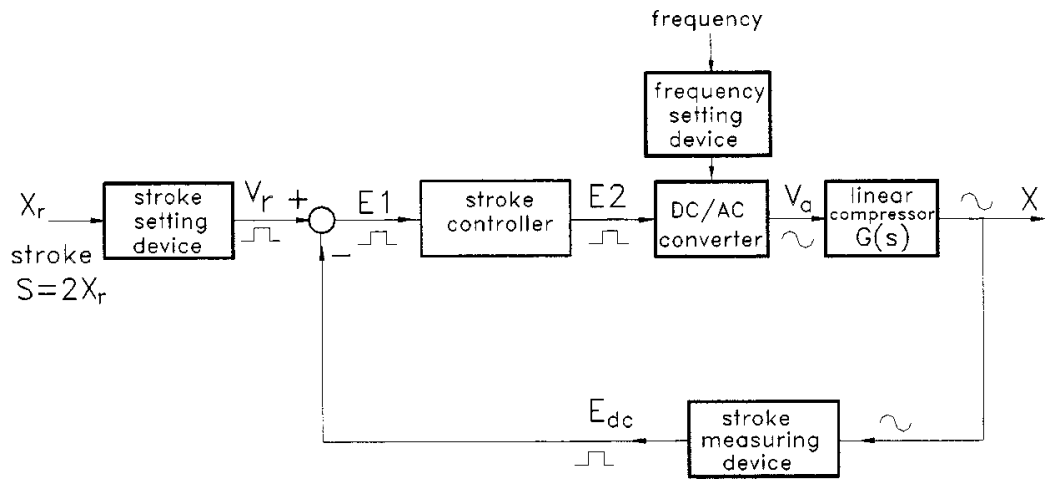

Fig. 6 Basic structure of control system

cludes some additional components as shown in Fig. 7. The bandwidth of $H(s)$ thus may change. For an accurate controller design, we performed another system identification experimentally for the controlled plant $H(s)$ using the aforementioned same technique and obtain the transfer function:

$$
H(s)=\frac{0.82}{s+5.824}
$$

Using critical damping design for the feedback system in order to reduce the overshot, we obtain a controller with $k_{i}=79.3$ and $K_{d 1}=5.95$, from a computer simulation using MATLAB.

Hardware Implementation and Test Results . The hardware design of the PDF controller is shown in Fig. 8. Two kinds of tests are performed: namely step response tests and disturbance rejection tests.

1 Step Response Test. The step response for an amplitude $\left(X_{r}\right)$ change from 0 to $4 \mathrm{~mm}$ is shown in Fig. 9. It is seen that it takes about $0.2 \mathrm{~s}$ to reach the steady state with a small tracking error in the amplitude or stroke of the piston. An error in the phase of the piston motion is present as compared to the simulation. As mentioned previously, the phase tracking is not important for many applications. The actual control signal E2 also agrees very well with the model prediction.

Several different step response tests were carried out. Figure 10 shows the step response for an amplitude change from $3 \mathrm{~mm}$ to 6 $\mathrm{mm}$. It takes about $0.2 \mathrm{~s}$ to reach steady state with small tracking error and having no overshoot. For an amplitude change from 6 $\mathrm{mm}$ to $3 \mathrm{~mm}$, it also takes about $0.2 \mathrm{~s}$ to reach steady state (see Fig. 11).
The control system can also be used to regulate the operating frequency of the linear compressor. Figure 12 shows that for a fixed amplitude $(5 \mathrm{~mm})$, the step response for a frequency change from $30 \mathrm{~Hz}$ to $47 \mathrm{~Hz}$ takes about $0.4 \mathrm{~s}$ to reach steady state with small tracking error. But, a slight overshoot in amplitude during the transient period occurs. Since the transient period is short $(0.4$ s) as compared to the transient performance of a refrigerator, the controller proves satisfactory.

2 Disturbance Rejection Test. A compressor may operate in a variable environment. Thus, a controller should be robust to disturbances. We applied a step disturbance signal $T_{d}$ inside the PDF controller (Fig. 7) and measured the response of the linear compressor. Figure 13 shows that at fixed amplitude $(5 \mathrm{~mm})$ and fixed frequency $(30 \mathrm{~Hz})$, the amplitude of the piston varies for a very short period of time (about $0.2 \mathrm{~s}$ ) right after the disturbance signal $(+4 \mathrm{~V})$ is applied. Though a variation in the amplitude is observed during this short period of time, the amplitude is still tracked very accurately at steady state. For a negative disturbance signal, the test results shown in Figure 14 reveals a similar trend. The performance proves again satisfactory.

Figure 15 shows the test results for a variable disturbance signal (positive change then negative change). The stroke variation is shown to remain small (within 10\%) and only occurs during a very short period of time (about $0.2 \mathrm{~s}$ ) right after the disturbance signal is applied. The above tests verify the robustness of the controller to disturbances.

\section{Discussion and Conclusion}

The system dynamics of a linear compressor is examined in the present study. A theoretical model was derived and a system iden-

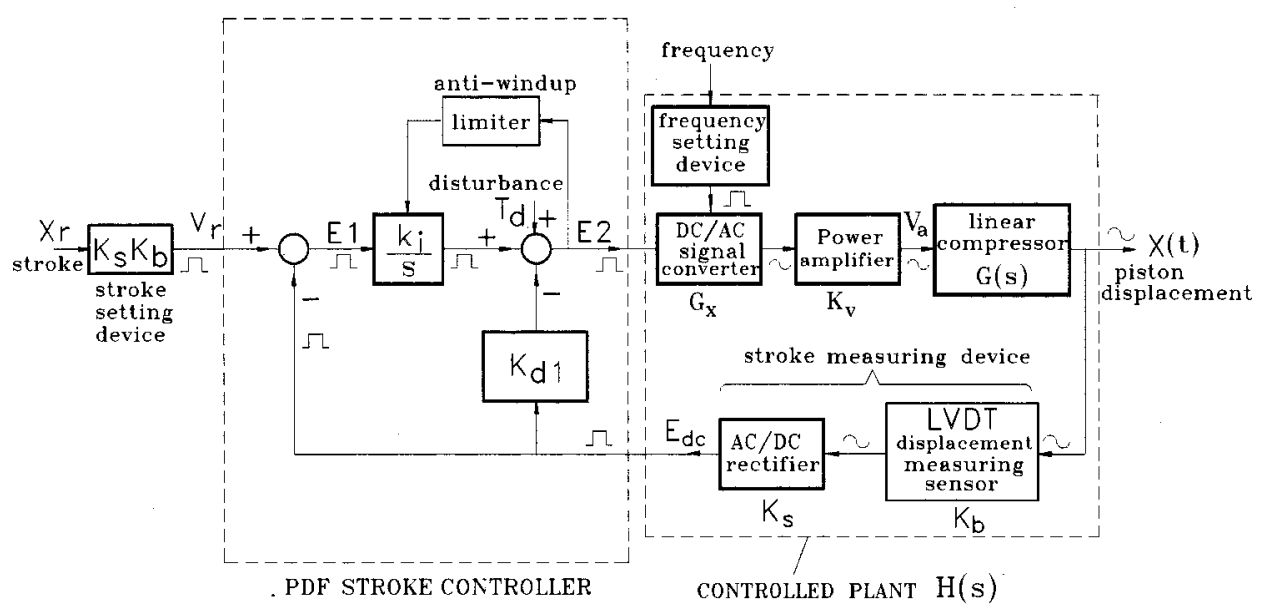

Fig. 7 PDF control system design 


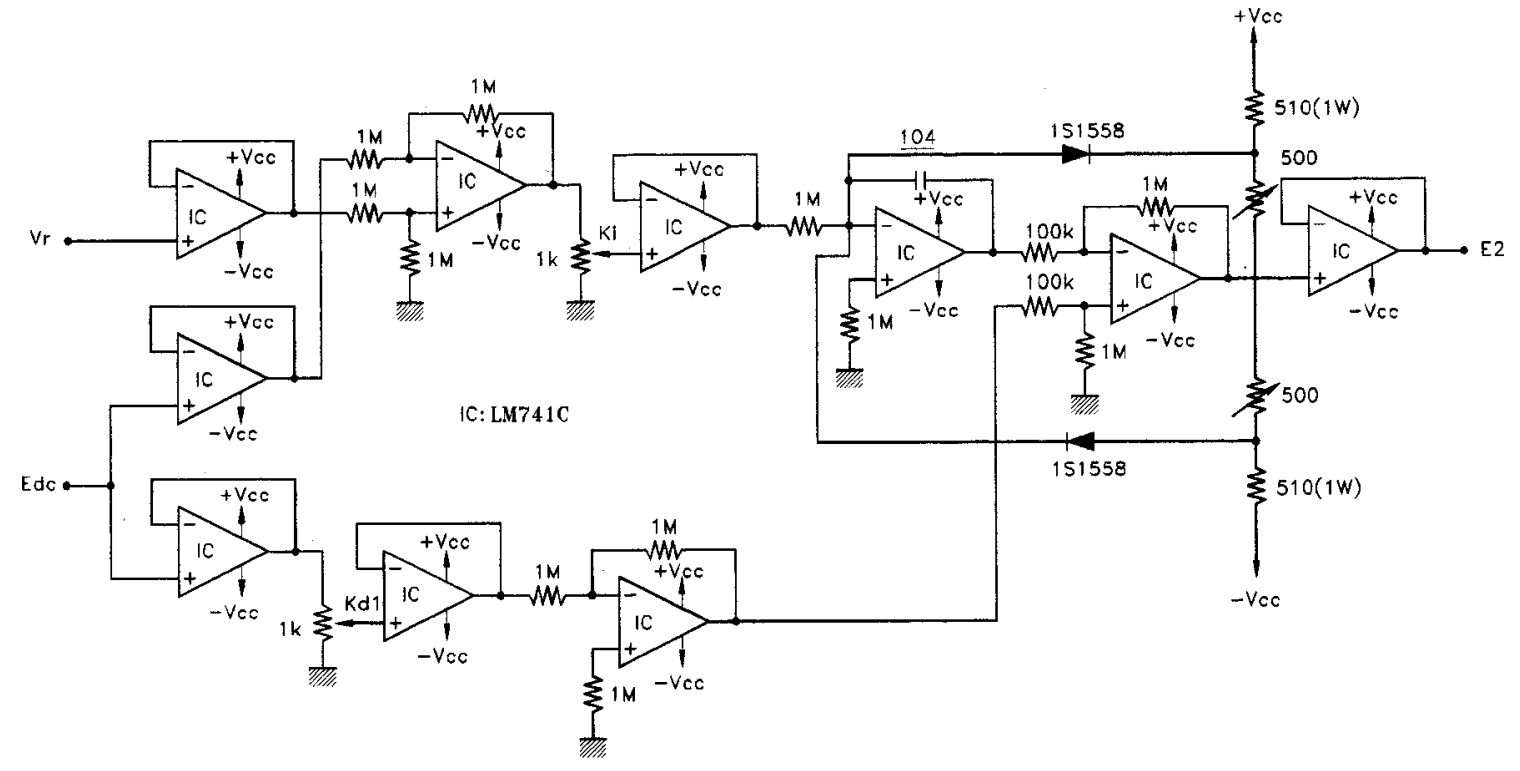

Fig. 8 Analog controller implementation
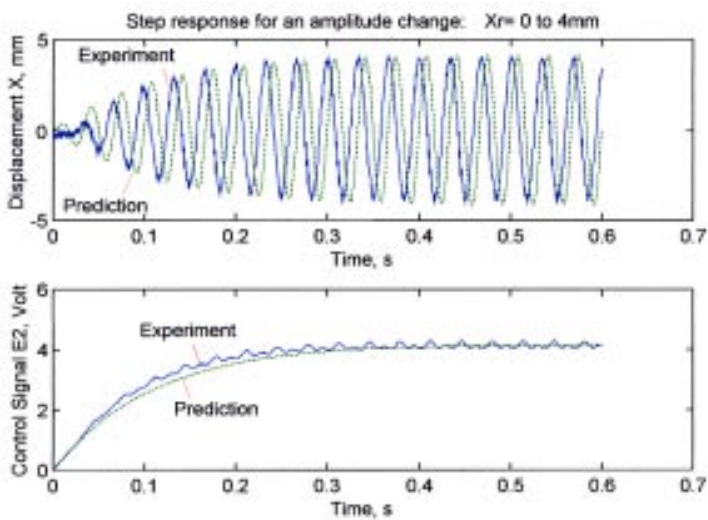

Fig. 9 Time response of displacement and control signal variation for amplitude change $\left(X_{r}=0 \rightarrow 4 \mathrm{~mm}\right)$
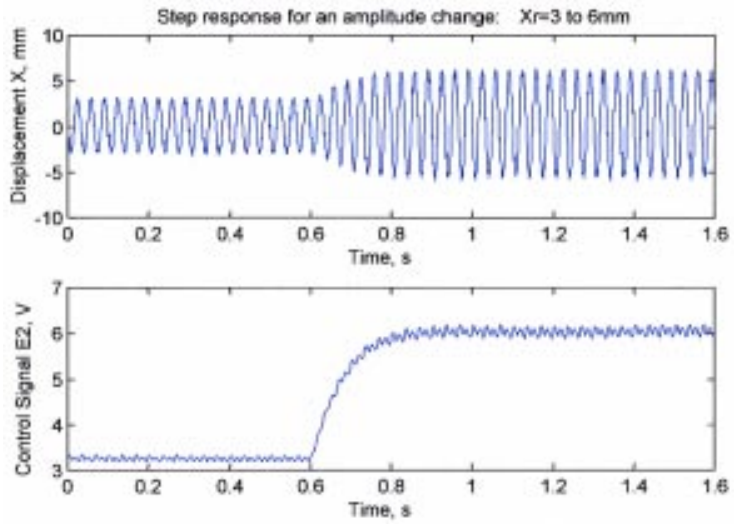

Fig. 10 Time response of displacement and control signal variation for amplitude change $\left(X_{r}=3 \mathrm{~mm} \rightarrow 6 \mathrm{~mm}\right)$
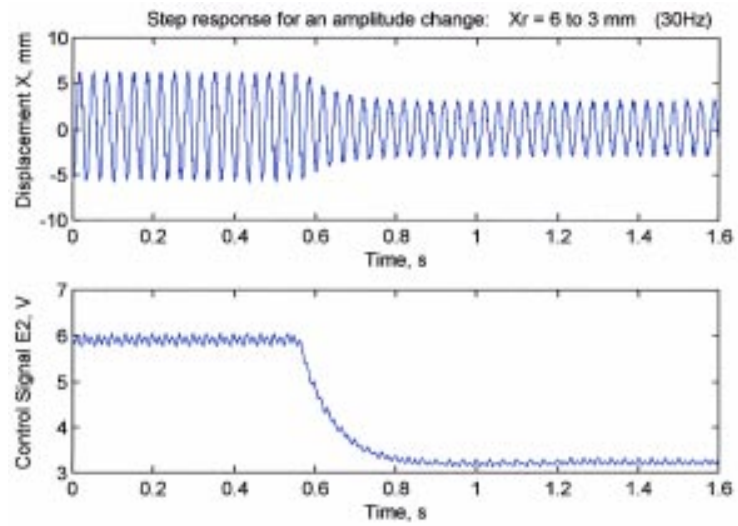

Fig. 11 Time response of displacement and control signal variation for amplitude change $\left(X_{r}=6 \mathrm{~mm} \rightarrow 3 \mathrm{~mm}\right)$
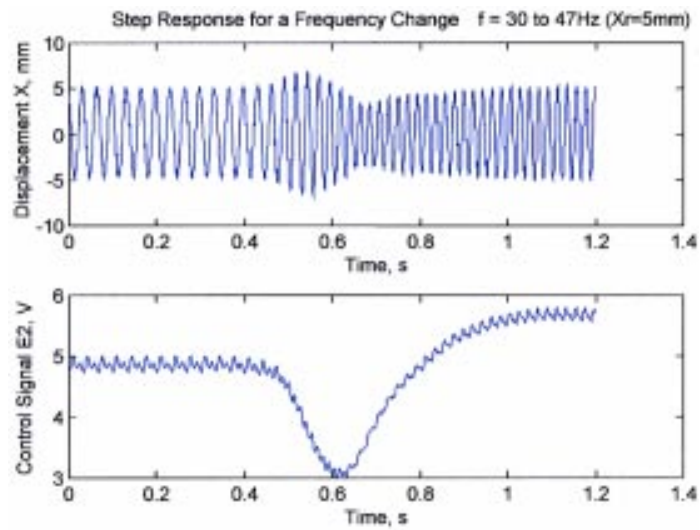

Fig. 12 Time response of displacement and control signal variation for frequency change $(f=30 \mathrm{~Hz} \rightarrow 47 \mathrm{~Hz})$

Transactions of the ASME 

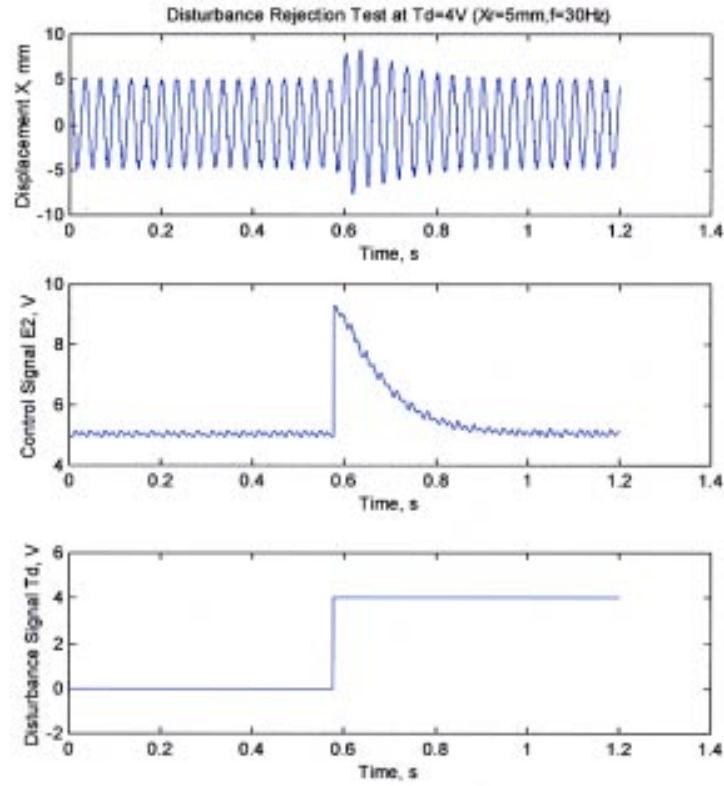

Fig. 13 Time response of displacement and control signal variation for step disturbance $(T d=+4 \mathrm{~V})$
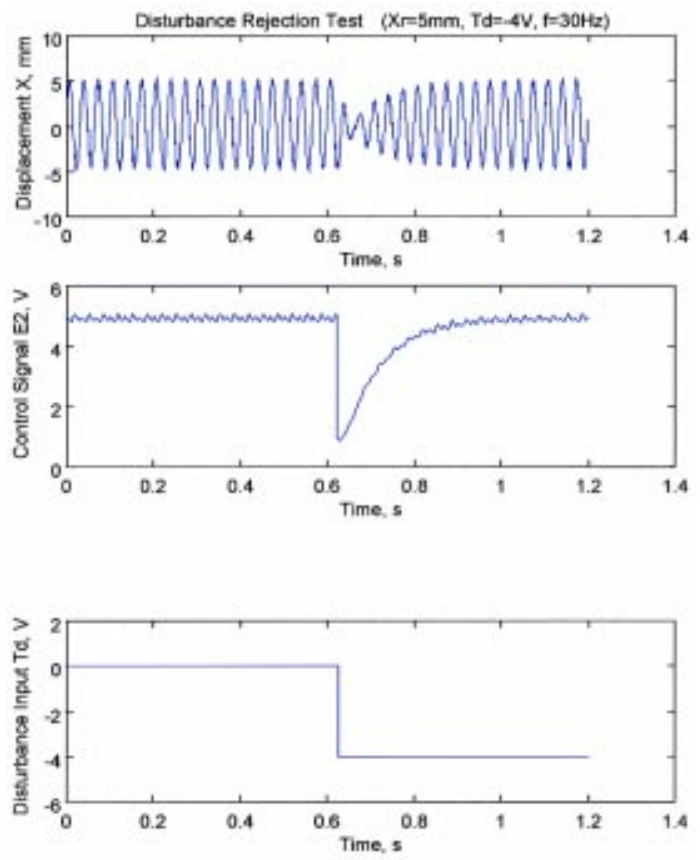

Fig. 14 Time response of displacement and control signal variation for step disturbance $(T d=-4 V)$

tification was performed experimentally. The experimental results agree with the theoretical model by letting $C=5 \mathrm{~N} \mathrm{~s} / \mathrm{m}$ and also show that the linear compressor behavior is linear over a wide range of applied voltage. A control system was developed based on the identified system dynamic model. A PDF controller is adopted. It is shown that the piston stroke can be controlled to a fixed value even with the presence of sudden isolated disturbances. Frequency can also be controlled so that the linear compressor can perform just like a variable-frequency compressor. The analog controller provides a means for fixing the piston
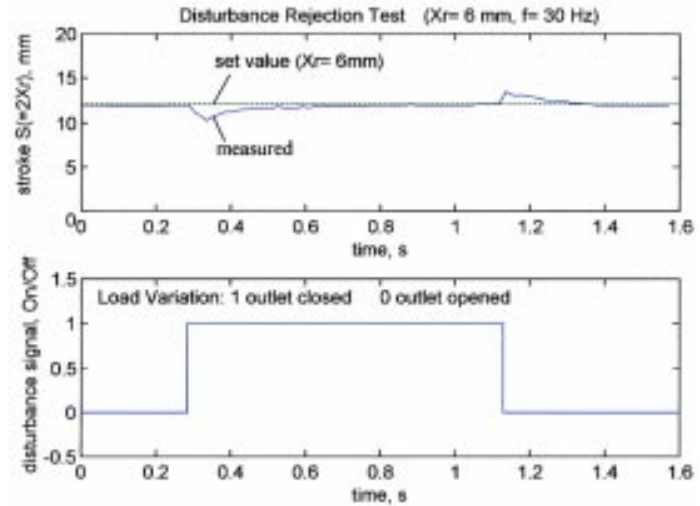

Fig. 15 Time response of stroke for variable disturbance

stroke of a linear compressor and thus it actually replaces the crank-shaft mechanism in a conventional reciprocating compressor.

Experimental results show that the performance of the control system is satisfactory, with fast response $(<0.3 \mathrm{~s})$, no overshoot $(<10 \%$ in stroke), accurate tracking, and good disturbance rejection. It is expected that the performance of a refrigerator or airconditioning system using a linear compressor can be improved if using the present controller for both stroke and frequency control. The present control system can also be employed in a variety of applications. The performance of air or water pumps using linear motor drives will be greatly improved by using the present control system. The pump will actually behaves as a metering pump.

\section{Acknowledgment}

The present study was supported by the National Science Council, Taiwan, ROC, through grant no. NSC84-2212-E002-004.

\section{Nomenclature}

$A_{p}=$ cross-sectional area of the piston, $\mathrm{m}^{2}$

$B=$ magnetic flux density Tesla

$C=$ viscous damping coefficient $\mathrm{N} \mathrm{s} / \mathrm{m}$

$I_{a}=$ driving current of linear motor, $A$

$R_{a}=$ resistance of the coil, Ohm

$K=$ spring constant $\mathrm{N} / \mathrm{m}$

$L_{a}=$ inductance of the coil, Henry

$l_{e}=$ effective length of the coil, $\mathrm{m}$

$M=$ equivalent mass of the moving coil and piston $\mathrm{kg}$

$\Delta P=$ pressure difference between the compression chamber and backside of the piston, $\mathrm{N} \mathrm{m}^{-2}$

$\mathrm{s}=$ Laplace variable, complex

$S=$ piston stroke $\left(=2 X_{r}\right), \mathrm{mm}$

$S_{X X}=$ auto-spectral density of $X(\mathrm{t})$

$S_{X V}=$ cross-spectral density of $X(\mathrm{t})$ and $V(\mathrm{t})$

$t=$ time, $s$

$V_{a}=$ driving voltage of linear motor, $\mathrm{V}$

$X=$ displacement of piston, $\mathrm{mm}$

\section{References}

[1] Cadman, R. V., and Cohen, R., 1969, "Electrodynamic Oscillating Compressors: Part 1-Design Based on Linearized Loads," ASME J. Basic Eng. Dec. pp. 656-663.

[2] Cadman, R. V. and Cohen, R., 1969, "Electrodynamic Oscillating Compressors: Part 2-Evaluation of Specific Designs for Gas Load," ASME J. Basic Eng., Dec., pp. 664-670.

[3] de Jonge, A. K., and Sereny, A., 1981, "Analysis and Optimization of a Linear Motor for the Compressor of a Cryogenic Refrigerator," Adv. Cryog. Eng., 27, pp. 631-640.

[4] Barry, R., 1992, "Linear Motor Driven Stirling Coolers for Military Commercial Applications," Proc IECEC, Vol. 5, pp. 5.149-5.154. 
[5] Stolfi, F. R., and Daniels, A., 1985, "Parametric Testing of a Linearly Driven Stirling Cryogenic Refrigerator," Proc. 3rd Int. Cryocooler Conf., pp. 80-98.

[6] Yang, Y. P., and Huang, B. J., 1998, "Fuzzy Control on the Phase and Stroke of a Linear Compressor of a Split-Stirling Cryocooler," Cryogenics, 38, pp. 231-238.

[7] Phelan, R. M., 1987, "Feedback Control Systems, privately published notes," Sibley School of Mechanical and Aeronautical Engineering, Cornell University, Ithaca, NY.
[8] Leu, M. C., and Freed, D. I., 1989, "PDF Subvariable Control and its Application to Robot Motion Control," ASME J. Dyn. Syst., Meas., Control, 111, pp. $452-461$.

[9] Kang, J. K, Lee, J. T., Kim, Y. M., Kwon, B. H., and Choi, K. S., 1991, “Speed Controller Design for Induction Motor Drives Using a PDF Control and Load Disturbance Observer," IECON Proceedings Industrial Electronics Conference, Vol. 1, pp. 799-803. 